\title{
Novos registros geográficos e variação na coloração de Dendropsophus pseudomeridianus (Amphibia, Anura, Hylidae) no Estado do Rio de Janeiro, Brasil
}

\author{
Adriano Lima Silveira* \\ Rafael Cunha Pontes \\ Rodrigo de Oliveira Lula Salles \\ Universidade Federal do Rio de Janeiro, Museu Nacional \\ Departamento de Vertebrados, Setor de Herpetologia \\ Quinta da Boa Vista, CEP 20940-040, Rio de Janeiro - RJ, Brasil \\ *Autor para correspondência \\ biosilveira@yahoo.com.br
}

Submetido em 30/11/2010

Aceito para publicação em 20/06/2011

\section{Resumo}

No presente trabalho são apresentados novos registros de Dendropsophus pseudomeridianus no Estado do Rio de Janeiro, Brasil, obtidos através de amostragens em campo em diversas áreas de Mata Atlântica no estado. Também são apresentadas variações na coloração da espécie. Dendropsophus pseudomeridianus foi coletada em localidades da baixada litorânea no norte do Rio de Janeiro e na região metropolitana da capital, em localidades mais interioranas no noroeste do estado, na Serra do Desengano e no vale do Paraíba do Sul na divisa com Minas Gerais. A espécie foi encontrada em brejos em áreas de floresta estacional semidecidual e floresta ombrófila densa. Os novos registros forneceram uma ampliação significativa da distribuição geográfica conhecida de D. pseudomeridianus, preencheram lacunas dessa distribuição e evidenciaram que a espécie é amplamente distribuída pelo estado. Os espécimes coletados exibiram variação na coloração, com dorso apresentando apenas pontuações e pequenas manchas marrons, barras oblíquas e mancha interocular ou extensa mancha em forma de "X", e o lábio superior ornamentado com pontuações, barras ou manchas circulares de cor branca.

Palavras-chave: Dendropsophus pseudomeridianus, Distribuição geográfica, Hylidae, Mata Atlântica, Rio de Janeiro

\section{Abstract}

New geographic records and color variation of Dendropsophus pseudomeridianus (Amphibia, Anura, Hylidae) in the State of Rio de Janeiro, Brazil. In this paper, we present the new records of Dendropsophus pseudomeridianus in the State of Rio de Janeiro, Brazil, obtained through field samplings in several areas of the Atlantic Rain Forest in that state. Herein are also presented the color variations of the species. Dendropsophus pseudomeridianus was collected in the costal lowlands of northern Rio de Janeiro and the capital's metropolitan region, in the state's northwest inland localities, in Serra do Desengano and Vale do Paraíba do Sul near the border with the state of Minas Gerais. This species was found in swamps in areas of semideciduous seasonal forest and dense ombrofilous forest. The new records provide a significant enhancement of the known geographic 
distribution of D. pseudomeridianus, filling in its gaps and providing evidence that the species is widely distributed throughout the state. The collected specimens exhibited color variation, dorsally they had only punctuations and small brown spots, oblique bars, and interocular stain or an extensive " $X$ " shaped stain, and the upper lip adorned with white color punctuations, bars, or circular spots.

Key words: Atlantic Rain Forest, Dendropsophus pseudomeridianus, Geographic distribution, Hylidae, Rio de Janeiro

Dendropsophus pseudomeridianus (Cruz, Caramaschi e Dias, 2000) é uma pequena espécie de hilídeo que habita áreas abertas e bordas de matas nos Estados do Rio de Janeiro e Espírito Santo, Brasil (CRUZ et al., 2000; IZECKSOHN; CARVALHO-E-SILVA, 2001). Na descrição original da espécie, sua distribuição geográfica foi definida como as regiões de baixada do Estado do Rio de Janeiro, a localidade tipo foi designada como o Município de Seropédica e foram apresentados registros também para os municípios de Araruama, Saquarema e Rio de Janeiro (Jacarepaguá) (CRUZ et al., 2000). Recentemente, D. pseudomeridianus foi registrada no Município de Mimoso do Sul, no sul do Estado do Espírito Santo (SILVA et al., 2008).

No presente trabalho, são apresentados novos registros de D. pseudomeridianus no Estado do Rio de Janeiro, a partir de amostragens realizadas em campo, no período de outubro de 2007 à janeiro de 2010. A maior parte dessas amostragens, realizadas no norte do estado, compuseram um extenso inventário de anuros realizado nas sub-bacias dos rios Muriaé, Imbé e Guaxindiba, compondo o Projeto de Gerenciamento Integrado de Agroecossistemas no Norte-Noroeste Fluminense - RIO RURAL/GEF, em realização pela Secretaria de Estado de Agricultura, Pecuária, Pesca e Abastecimento (SEAPPA) do Governo do Estado do Rio de Janeiro. Os exemplares coletados foram depositados como material testemunho na Coleção de Anfíbios do Museu Nacional, Universidade Federal do Rio de Janeiro (MNRJ).

As coletas foram conduzidas mediante a Autorização para Atividades com Finalidade Científica no 14590-1, expedida pelo Instituto Brasileiro do Meio Ambiente e dos Recursos Naturais Renováveis (IBAMA), e a Autorização para Pesquisa Científica IEF/ RJ № 013/2008 (específica para a Estação Ecológica de Guaxindiba), expedida pela Fundação Instituto Estadual de Florestas do Rio de Janeiro (atualmente Instituto Estadual do Ambiente - INEA).

Exemplares adultos de D. pseudomeridianus (Figura 1) foram coletados em oito municípios do Estado do Rio de Janeiro: Campos dos Goytacazes (Lagoa de Cima); Itaperuna (Fazenda São Vicente e ao longo da rodovia RJ 121, entre Natividade e o Distrito de Raposo); Magé (Ponte Preta); Niterói (Serra da Tiririca); Santa Maria Madalena (Serra do Desengano: Rancho da Lelê e nas proximidades do centro da cidade); São Francisco do Itabapoana (Estação Ecológica de Guaxindiba e entorno); Três Rios (brejos próximos ao rio Paraibuna) (Figura 2, Anexo 1).

Os novos registros ampliam de forma significativa a distribuição geográfica conhecida de D. pseudomeridianus para todo o norte fluminense, incluindo a baixada litorânea, a região mais interiorana no noroeste do estado e a Serra do Desengano no norte da Serra do Mar, e para a região interiorana no vale do rio Paraíba do Sul na divisa com o Estado de Minas Gerais, além de dois registros adicionais na região metropolitana do Rio de Janeiro. Desta forma, é preenchida a lacuna de distribuição entre o litoral central do Estado do Rio de Janeiro e o sul do Espírito Santo.

A espécie foi registrada em diversos ambientes de Mata Atlântica (senso IBGE, 2004). Em Santa Maria Madalena, Duque de Caxias e Niterói, $D$. pseudomeridianus foi registrada em brejos em área de floresta ombrófila densa, incluindo região serrana na primeira localidade (Serra do Desengano) e baixada litorânea nas demais. Na Estação Ecológica de Guaxindiba, em Itaperuna, Lagoa de Cima e Três Rios, a espécie foi registrada em brejos em área de floresta estacional semidecidual, além da margem de uma grande lagoa natural (Lagoa de Cima). 


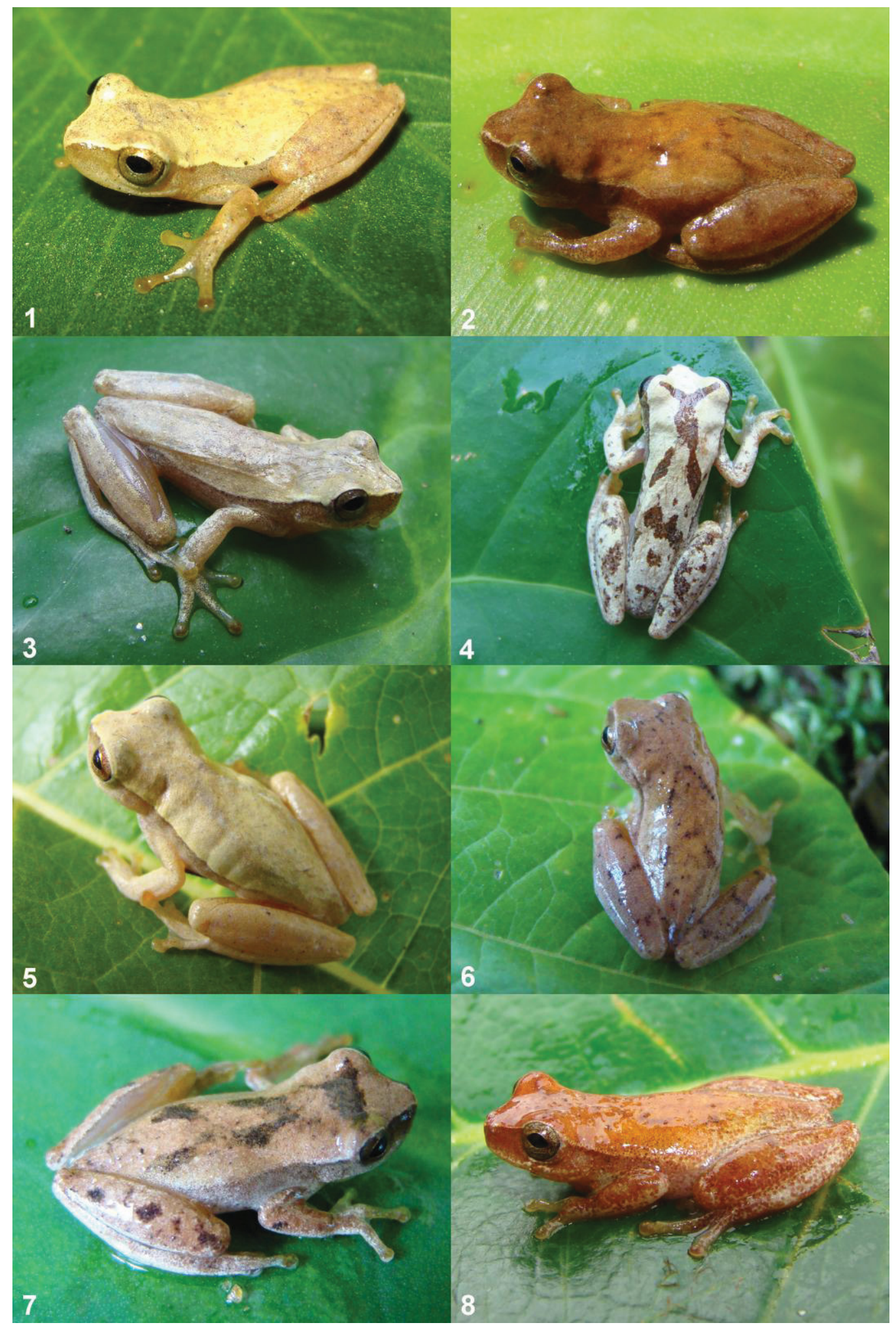

FIGURA 1: Exemplares de Dendropsophus pseudomeridianus coletados no Estado do Rio de Janeiro. Procedências: 1) Ponte Preta, Magé; 2) Fazenda São Vicente, Itaperuna; 3 e 4) Lagoa de Cima, Campos dos Goytacazes; 5 a 8) Rancho da Lelê, Santa Maria Madalena (Serra do Desengano). 


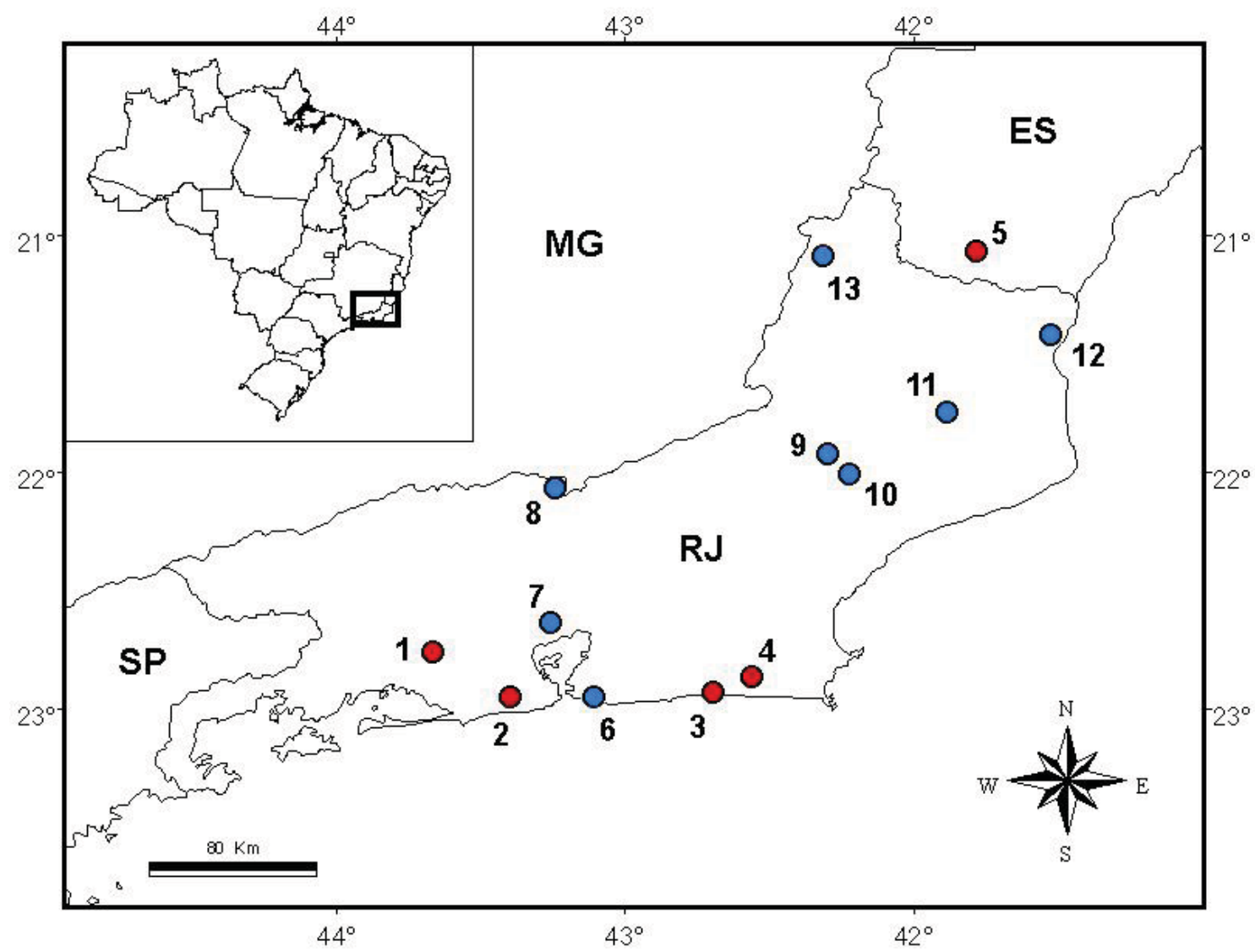

FIGURA 2: Distribuição geográfica conhecida de Dendropsophus pseudomeridianus. Registros prévios em literatura (pontos vermelhos): Estado do Rio de Janeiro: 1) Seropédica (localidade-tipo); 2) Jacarepaguá, Rio de Janeiro; 3) Saquarema; 4) Araruama; Estado do Espírito Santo: 5) Mimoso do Sul (CRUZ et al., 2000; SILVA et al., 2008). Novos registros no Estado do Rio de Janeiro (pontos azuis): 6) Serra da Tiririca, Niterói; 7) Ponte Preta, Magé; 8) brejos próximos ao rio Paraibuna, Três Rios; 9) proximidades da cidade, Santa Maria Madalena (Serra do Desengano); 10) Rancho da Lelê, Santa Maria Madalena (Serra do Desengano); 11) Lagoa de Cima, Campos dos Goytacazes; 12) Estação Ecológica de Guaxindiba e entorno, São Francisco do Itabapoana; 13) Fazenda São Vicente e margem da rodovia RJ 121 (entre Natividade e o Distrito de Raposo), Itaperuna.

Todos os exemplares coletados apresentaram os caracteres diagnósticos de $D$. pseudomeridianus, segundo CRUZ et al. (2000), exceto algumas variações quanto à coloração. De acordo com a descrição da espécie, $D$. pseudomeridianus não apresenta faixas longitudinais no dorso do corpo, o qual é pintalgado por pontos esparsos e pequenas concrescências marrom-escuro. Este padrão foi observado em espécimes de todas as localidades de registros da espécie (presente estudo), mas em Santa Maria Madalena e em Lagoa de Cima alguns exemplares apresentaram dorso com os pontos esparsos, uma mancha interocular geralmente em forma de "V" e dois pares de barras oblíquas, de cor marrom escuro. Essas manchas exibiram variação de muito delgadas a largas e irregulares, e as barras do par anterior sendo mais compridas e podendo estar unidas entre si medialmente e unidas à mancha interocular, neste caso formando uma grande mancha em forma de "X" (Figura 1). Variação semelhante na coloração do dorso foi descrita para $D$. cruzi (POMBAL JR.; BASTOS, 1998) no Brasil central, com exemplares exibindo dorso sem desenho, com manchas irregulares, com duas faixas largas ou com desenho em forma de " $X$ " (POMBAL JR.; BASTOS, 1998).

Mancha marrom dorsal em forma de "X" é um caractere diagnóstico de $D$. berthalutzae (Bokermann,1962) (CRUZ et al., 2000), espécie ocorrente 
no Estado do Rio de Janeiro e que foi registrada em simpatria com D. pseudomeridianus em Santa Maria Madalena (brejo no Rancho da Lelê), no presente estudo, em 16 de março de 2008 (MNRJ 53242-49; Figura 3). Entretanto os exemplares coletados de $D$. berthalutzae também apresentaram cabeça mais comprida e mais larga em relação ao tronco, focinho mais comprido em relação à cabeça em vista dorsal, prega supratimpânica desenvolvida, olhos mais proeminentes, maior constrição na região imediatamente posterior à cabeça em vista dorsal, coloração do flanco semelhante à do dorso e uma estreita e discreta linha marrom médio-dorsal geralmente presente. Por outro lado, os exemplares de D. pseudomeridianus coletados apresentaram cabeça mais curta e mais estreita em relação ao tronco, focinho mais curto em relação à cabeça, prega supratimpânica não desenvolvida, olhos menos proeminentes, menor constrição na região posterior à cabeça, flanco mais escuro que o dorso e ausência de linha médio-dorsal, sendo então diferenciados de $D$. berthalutzae.

As machas no dorso encontradas em $D$. pseudomeridianus poderiam gerar confusão com $D$. meridianus (B. Lutz, 1954); mas, esta espécie exibe faixas longitudinais escuras no dorso e extremidade do focinho sem protuberância (CRUZ et al., 2000; Figura 3), ao passo que $D$. pseudomeridianus exibe barras oblíquas, as quais podem se unir formando um " $X$ ", mas não chegam a caracterizar faixas longitudinais, e apresenta também uma protuberância na extremidade do focinho.

Outra variação observada foi em relação à coloração da lateral da cabeça. Segundo a descrição da espécie, D. pseudomeridianus apresenta região loreal marrom e não exibe manchas brancas sob os olhos, característica esta diagnóstica de D. branneri (Cochran, 1948) e D. werneri (Cochran, 1952) (Figura 3) (CRUZ et al.,

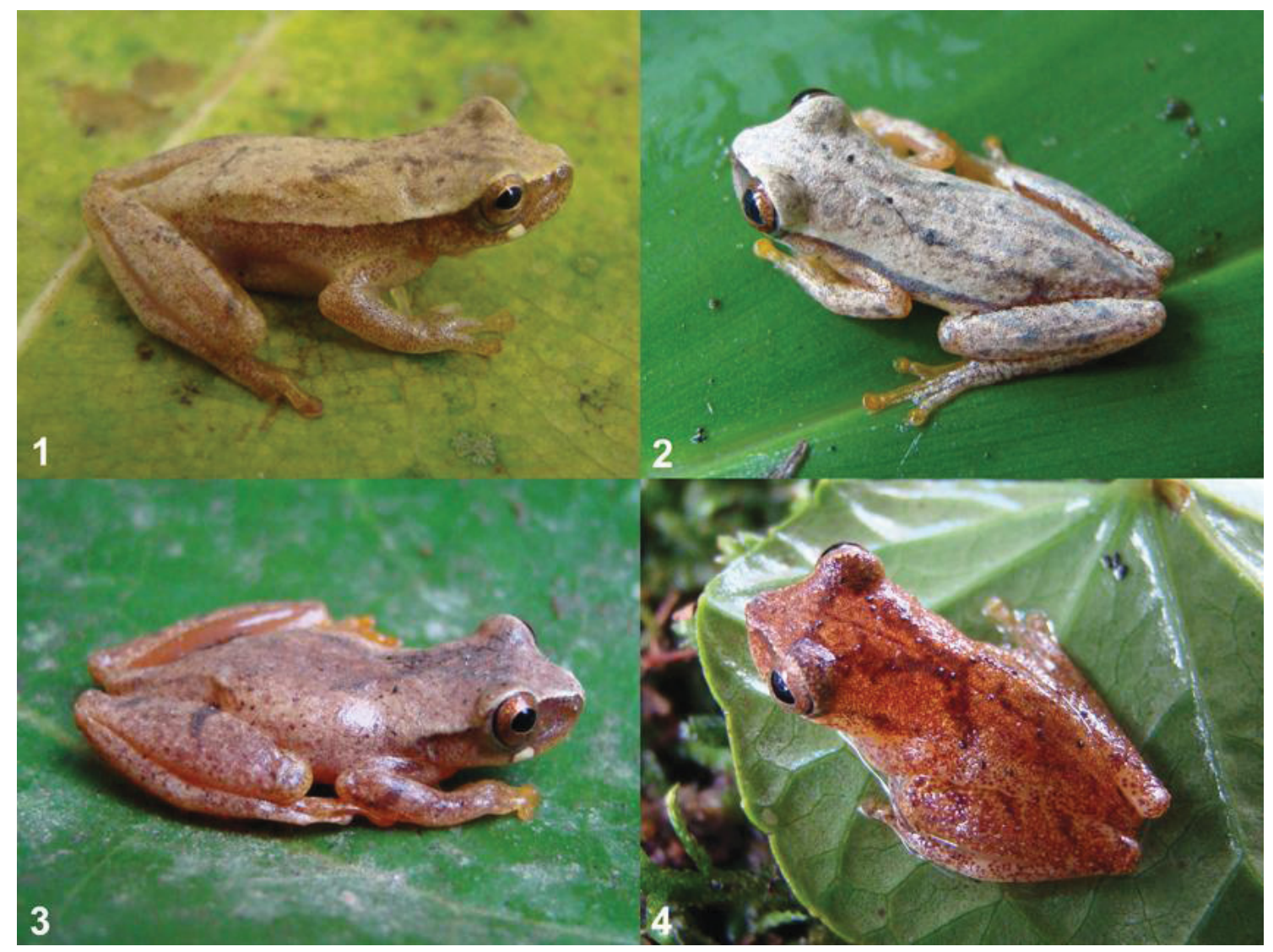

FIGURA 3: Exemplares das espécies que apresentam coloração mais semelhante a Dendropsophus pseudomeridianus: 1) D. branneri, coletado em Cabo de Santo Agostinho, PE; 2) D. meridianus, coletado em Duque de Caxias, RJ; 3) D. werneri, coletado em Curupá, SC e 4) D. berthalutzae, coletado em Santa Maria Madalena na Serra do Desengano, RJ. 
2000). Entretanto, os exemplares coletados de $D$. pseudomeridianus (presente estudo) exibiram a lateral da cabeça marrom, com pontuações brancas na borda do lábio superior, variando de raras e esparsas a abundantes $\mathrm{e}$ aglomeradas, podendo formar manchas brancas alongadas ou, raramente, arredondadas, abaixo do olho. A frequência de ocorrência de manchas brancas arredondadas foi variável entre as localidades de coleta, estando presentes em exemplares de Santa Maria Madalena, Lagoa de Cima e Itaperuna e ausentes em exemplares da Estação Ecológica de Guaxindiba.

Os espécimes aqui identificados como $D$. pseudomeridianus foram diferenciados de D. branneri por apresentarem canto rostral bem marcado e focinho mais largo, e diferenciados de $D$. werneri por apresentarem narinas salientes; ao passo que $D$. branneri apresenta canto rostral pouco marcado e focinho mais estreito e curto, e D. werneri apresenta narinas não salientes (CRUZ et al., 2000).

Por apresentarem mancha branca arredondada sob o olho, alguns exemplares de D. pseudomeridianus procedentes do Rio de Janeiro podem ser confundidos com D. branneri. De fato, nas amostragens realizadas no presente estudo, as quais abrangeram grande área do Estado do Rio de Janeiro, não se registrou a presença de D. branneri e é provável que exemplares procedentes do estado até então identificados como $D$. branneri tratem-se de espécimes de D. pseudomeridianus com mancha branca subocular (BASTOS; POMBAL JR., 1996; ROCHA et al., 2004; LUTZ, 1973). Neste caso, é possível que $D$. branneri não ocorra no Rio de Janeiro.

Os exemplares de D. branneri analisados por CRUZ et al. (2000) procedem dos estados da Bahia e Alagoas (a norte do Rio de Janeiro), ao passo que os espécimes de $D$. werneri procedem de São Paulo e Santa Catarina (a sul do Rio de Janeiro). Assim, provavelmente $D$. pseudomeridianus ocorre em área intermediária às áreas de distribuição de $D$. branneri e $D$. werneri.

\section{Agradecimentos}

A Gesinaldo Moura da Silva, pelo auxílio nas coletas em campo, a Carlos Alberto Cruz pelo auxílio na identificação taxonômica, à SEAPPA, pelo suporte às atividades de campo, e ao IBAMA e INEA, pela concessão de autorização para coleta de anfíbios.

\section{Referências}

BASTOS, R. P.; POMBAL JR., J. P. A new species of Hyla (Anura: Hylidae) from eastern Brazil. Amphibia-Reptilia, Leiden, v. 17, p. 325-331, 1996.

CRUZ, C. A.; CARAMASCHI, U; DIAS, A. G. Espécie nova de Hyla Laurent, 1768 do Estado do Rio de Janeiro, Brasil. Boletim do Museu Nacional, Nova Série, Zoologia, Rio de Janeiro, v. 434, p. $1-8,2000$.

IBGE - INSTITUTO BRASILEIRO DE GEOGRAFIA E ESTATÍSTICA. Mapa de Vegetação do Brasil. 3. ed. [S.1.]: Instituto Brasileiro de Geografia e Estatística, 2004. 1 mapa. Escala: 1:5.000.000

IZECKSOHN, E.; CARVALHO-E-SILVA, S. P. Anfíbios do Município do Rio de Janeiro. Rio de Janeiro: Editora UFRJ, 2001. $148 \mathrm{p}$.

LUTZ, B. Brazilian Species of Hyla. Austin: University of Texas Press, 1973. $260 \mathrm{p}$.

POMBAL JR, J. P.; BASTOS, R. P. Nova espécie de Hyla Laurenti, 1768 do Centro-Oeste Brasileiro e a posição taxonômica de $H$. microcephala werneri Cochran, 1952 e H. microcephala meridiana B. Lutz, 1952 (Anura, Hylidae). Boletim do Museu Nacional, Nova Série, Zoologia, Rio de Janeiro, n. 390, p. 1-14, 1998.

ROCHA, C. F. D.; BERGALlO, H. G.; POMBAL JR., J. P.; GEISE, L.; SLUYS, M. V.; FERNANDES, R.; CARAMASCHI, U. Fauna de anfíbios, répteis e mamíferos do Estado do Rio de Janeiro, Sudeste do Brasil. Publicações Avulsas do Museu Nacional, Rio de Janeiro, n. 104, p. 1-24, 2004.

SILVA, G. R.; CARVALHO-E-SILVA, S. P.; CARVALHO-ESILVA, A. M. P. T. Notes on geographic distribution: Amphibia, Anura, Hylidae, Dendropsophus pseudomeridianus: Distribution extension and geographic distribution map. Check List, Campinas, v. 4 , n. 1 , p. $15-17,2008$. 


\section{Anexo 1}

Espécimes testemunhos de Dendropsophus pseudomeridianus coletados no presente estudo e respectivas procedências com coordenadas geográficas e datas de coleta. MNRJ - Coleção de Anfíbios do Museu Nacional, Universidade Federal do Rio de Janeiro.

ESTADO DO RIO DE JANEIRO: MUNICÍPIO DE CAMPOS DOS GOYTACAZES: Lagoa de Cima:

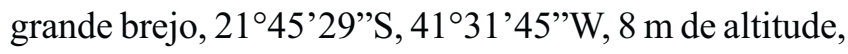
coletado em 05/04/2008: MNRJ 52596-52602; pequeno brejo, 21 ${ }^{\circ} 46^{\prime} 16^{\prime}$ 'S, $41^{\circ} 32^{\prime} 08^{\prime \prime} \mathrm{W}, 16 \mathrm{~m}, 05 / 04 / 2008$ : MNRJ 52603-52604; brejo, 21 ${ }^{\circ} 44^{\prime} 47^{\prime \prime} \mathrm{S}, 41^{\circ} 29^{\prime} 50^{\prime \prime} \mathrm{W}$, 6 m, 06/04/2008: MNRJ 52575-52576; grande brejo, 2144'07'S, 41 29 '41'W, 7 m, 06/04/2008: MNRJ 52591-52595; brejo, 21 ${ }^{\circ} 45^{\prime} 06^{\prime} \mathrm{S}, 41^{\circ} 32^{\prime} 39^{\prime \prime} \mathrm{W}, 23 \mathrm{~m}$, 07/04/2008: MNRJ 52605-52620. MUNICÍPIO DE

ITAPERUNA: brejo na margem da rodovia RJ 214 entre Natividade e o Distrito de Raposo (Itaperuna), $21^{\circ} 05^{\prime} 20$ "S, 42 03 ' $15^{\prime \prime} \mathrm{W}, 219$ m, 27/03/2008: MNRJ 52686-52689; brejo na margem da rodovia RJ 214, $21^{\circ} 05^{\prime} 13$ 'S, 42॰02'05'”, $227 \mathrm{~m}, 28 / 03 / 2008$ : MNRJ 52679; Fazenda São Vicente: brejo, $21^{\circ} 05^{\prime} 09^{\prime}$ 'S, 42 01 '35”'W, $212 \mathrm{~m}, 28 / 03 / 2008$ : MNRJ 5268052685. MUNICÍPIO DE MAGÉ: Ponte Preta, $6^{\circ}$
Distrito (Piabetá), divisa com o Município de Duque de Caxias, 22³8'10'S, 4311'28'W, 22 m, 30/01/2010: MNRJ 71120-71127. MUNICÍPIO DE NITERÓI: Serra da Tiririca, Engenho do Mato, 22 $57^{\circ} 15^{\prime \prime} \mathrm{S}$, 4301'20", 25 m, 18/11/2009: MNRJ 62301-62303. MUNICÍPIO DE SANTA MARIA MADALENA: brejo próximo à cidade, $21^{\circ} 55^{\prime} 36^{\prime}$ 'S, $42^{\circ} 01^{\prime} 01^{\prime}$ ”W, 435 m, 15/03/2008: MNRJ 52585-52590; Rancho da Lelê, brejo, 22 $2^{\circ} 00^{\prime} 42^{\prime \prime S}, 41^{\circ} 55^{\prime} 22^{\prime \prime} \mathrm{W}, 443 \mathrm{~m}, 16 / 03 / 2008$ : MNRJ 52579-52581, 52583-52584; Rancho da Lelê,

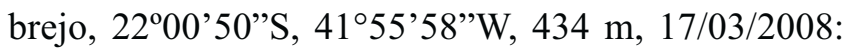
MNRJ 52577-52578, 52582. MUNICÍPIO DE SÃO FRANCISCO DO ITABAPOANA: Estação Ecológica de Guaxindiba: brejo na borda da Mata do Carvão,

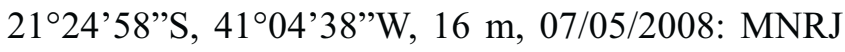
52568-52574; Estação Ecológica de Guaxindiba: poça próxima à Mata do Carvão, $21^{\circ} 25^{\prime} 45^{\prime}$ ”S, 41 ${ }^{\circ} 04^{\prime} 56^{\prime}$ ”W, 11 m, 09/05/2008: MNRJ 52557-52559; Fazenda São Pedro de Alcântara II: brejo em canavial próximo à Estação

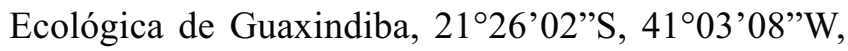
7 m, 07/05/2008: MNRJ 52560-52567. MUNICÍPIO DE TRÊS RIOS: brejo próximo ao rio Paraibuna, 2203'57'S, 4310'01'W, 285m, 25/10/2007: MNRJ 50399-50403; brejo próximo ao rio Paraibuna, 22 04 '04"S, 4310'26”'W, 305 m, 26/10/2007: MNRJ 50361-50365. 